\title{
Microwave Processing for Advanced Electro-optic Materials
}

\author{
CRADA No. ORNL96-0478
}

\section{Final Report}

\author{
PI: L. A. Boatner, Oak Ridge National Laboratory, Solid State Division
}

PARTICIPANT : Commercial Crystal Laboratories (CCL), Naples, Florida

\section{Project Summary:}

This project addressed the technical and scientific goals of developing new methods for the formation of striation-free single crystals of potassium tantalate niobate. This solid-solution system has the potential for serving as a general electro-optic material with a wide range of optical applications. The performance of the material is, however, severely limited by the effects of compositional inhomogeneity that is generally induced during the single crystal growth process due to the nature of the binary phase diagram of the mixed tantalate/niobate system. Single-crystal boules of potassium tantalate niobate $\left(\mathrm{KTa}_{1-\mathrm{x}} \mathrm{Nb}_{\mathrm{x}} \mathrm{O}_{3}\right.$ or KTN) with varying tantalum-to-niobium ratios (or values of $\mathrm{x}$ ) were grown under a variety of experimental conditions. The resulting single crystals were characterized in terms of their compositional homogeneity and optical quality. Single crystals were grown using both the most-favorable established set of growth parameters as well as in the presence of programmed oscillatory temperature variations. The purpose of these deliberately induced variations was to introduce controlled compositional variations and associated optical striations in the solid-solution single crystals. The overall objective of the effort was to utilize microwave heating and processing methods to treat the inhomogeneous single crystals for the purpose of eliminating the compositional variations that lead to striations and the associated varying changes in the refractive index of the material. In order to realize the ultimate goal of the effort, 
it was necessary to develop methods that would lead to the effective coupling of the microwave field to the KTN single crystals. Achieving the technical and commercial goals of this effort would have made it possible to introduce an important new electro-optic product into the market place, to improve our fundamental understanding of solid-state diffusion processes in general (and of microwave-assisted thermal processes in particular), and finally, to create a new class of industrial applications for microwave heating.

\section{Project Background}

Electro-optic materials in the mixed-solid-solution system potassium tantalatepotassium niobate (KTN) have electro-optic coefficients, refractive indices, and other physical properties that make these materials superior candidates for numerous advanced optical and opto-electronic applications. The devices in which single-crystal KTN can be utilized (e.g., optical mixers, phase-conjugate mirrors, optical modulators, switches, and couplers) are applicable to the monitoring and control of energy-related devices, to systems for environmental monitoring, and in optical sensing and communication systems for nationalsecurity-type applications. The penetration of KTN into the mainstream electrooptic marketplace has traditionally been limited by the difficulty of growing large homogeneous single-crystals. Homogeneity is a central issue since the growth of KTN single crystals since the growth process involves the solidification of a binary solid solution. Since such systems do not solidify congruently, minute temperature fluctuations at the growing solid/liquid interface create compositional fluctuations that are manifested in the form of deleterious optical striations throughout the boule. There were indications that microwave heating processes could create "non-thermal" effects, i.e., enhanced rates of diffusion, that could be exploited in the homogenization of KTN crystals thereby making this promising material a viable important commercial product. Compositionally striated KTN crystals that could be treated under controlled conditions of microwave frequency, power, and temperature offered the potential for developing a "breakthrough" technology. This breakthrough would have provided a new material for the electro-optics community. Additionally KTN represents an excellent model system for studying and understanding diffusion in microwave-assisted heating processes. 


\section{Scientific \& Technical Progress:}

KTN single-crystal boules were grown and extensively characterized with respect to their spatial compositional profiles and associated optical quality. This optical characterization included evaluations of the performance of the material in the mode of four-wave-mixing. An optimized process for the growth of large, highoptical-quality single crystals of KTN with any given desired tantalum-to-niobium ratio was developed based on the combined experimental and analytical findings. The KTN single-crystal boules were thermally processed at different temperatures and under different atmospheric conditions by using conventional heating. The processing variables that were investigated included the specimen temperature, treatment atmospheres, and annealing times. Numerous studies of a wide range of sample configurations and microwave heating-system parameters were investigated in efforts to couple the microwave energy effectively to the single crystal potassium tantalate samples. Potassium tantalate niobate is, however, characterized by a very high dielectric constant. This high dielectric constant proved to be an insurmountable barrier in regard to achieving a configuration where microwave energy could be used to process the mixed solid-solution crystals. Ultimately, only conventional heating using resistance furnaces could be applied to treating the striated single crystals. This conventional approach proved to be relatively ineffective in removing the undesirable striations. Nevertheless, significant progress was made in our ability to grow single crystals of $\mathrm{KTN}$ and of the pure end member $\mathrm{KTaO}_{3}$ with improved crystal quality. These improved materials have been used in the characterization experiments noted above and in the development of new applications of these materials - including their use as substrates for the epitaxial growth of ferroelectric thin films. Examples of some of the results issuing from this effort are described in our publications as listed below. 


\section{Resulting Publications:}

1. H. Liu, F. Fernandez, W. Jia, and L.A. Boatner, "Transient Grating in a $\mathrm{KNbO}_{3} / \mathrm{KTaO}_{3}$ Superlattice," p. 182-187 in Optical Diagnostic Methods for Inorganic Transmissive Materials, ed. by R. U. Datla and L. M. Hanssen, Proceedings of the SPIE, Vol. 3425 (1998).

2. E. D. Specht, H.-M. Christen, D. P. Norton, and L. A. Boatner, "X-Ray Diffraction Measurement of the Effect of Layer Thickness on the Ferroelectric Transition in Epitaxial $\mathrm{KTaO}_{3} / \mathrm{KNbO}_{3}$ Multilayers," Phys. Rev. Lett. 80, (19) 4317-4320 (1998).

3. H.-M. Christen, E. D. Specht, D. P. Norton, M. F. Chisholm, and L. A. Boatner, "LongRange Ferroelectric Interactions in $\mathrm{KTaO}_{3} / \mathrm{KNbO}_{3}$ Superlattice Structures," Appl. Phys. Lett. 72, (20) 2535-2537 (1998).

4. H.-M. Christen, D. P. Norton, L. A. Gea, and L. A. Boatner, "Pulsed Laser Deposition of Solid-Solution Films Using Segmented Targets," Thin Solid Films 312, 156-159 (1998).

5. H.-M. Christen, L. A. Boatner, L. Q. English, L. A. Gea, P. J. Marrero, and D. P. Norton, "Pulsed Laser Deposition of Epitaxial $\operatorname{Sr}\left(\mathrm{Ru}_{\mathrm{x}} \mathrm{Sn}_{1-\mathrm{x}}\right) \mathrm{O}_{3}$ Thin Film Electrodes and $\mathrm{KNbO}_{3} / \mathrm{Sr}\left(\mathrm{Ru}_{\mathrm{x}} \mathrm{Sn}_{1-\mathrm{x}}\right) \mathrm{O}_{3}$ Bilayers," p. 203-207 in Epitaxial Oxide Thin Films, ed. by D. K. Fork et al., Mat. Res. Soc. Symp. Proc., Vol. 401 (Materials Research Society, Pittsburgh, Pennsylvania, 1996).

6. Y. Kim, A. Erbil, and L. A. Boatner, "Substrate Dependence in the Growth of Epitaxial $\mathrm{Pb}_{1-x} \mathrm{La}_{x} \mathrm{TiO}_{3}$ Thin Films," Appl. Phys. Lett. 69, (15) 2187-2189 (1996).

7. H.-M. Christen, L. A. Boatner, J. D. Budai, M. F. Chisholm, L. A. Gea, P. J. Marrero, and D. P. Norton, "The Growth and Properties of Epitaxial $\mathrm{KNbO}_{3}$ Thin Films and $\mathrm{KNbO}_{3} / \mathrm{KTaO}_{3}$ Superlattices," Appl. Phys. Lett. 68, (11) 1488-1490 (1996). 


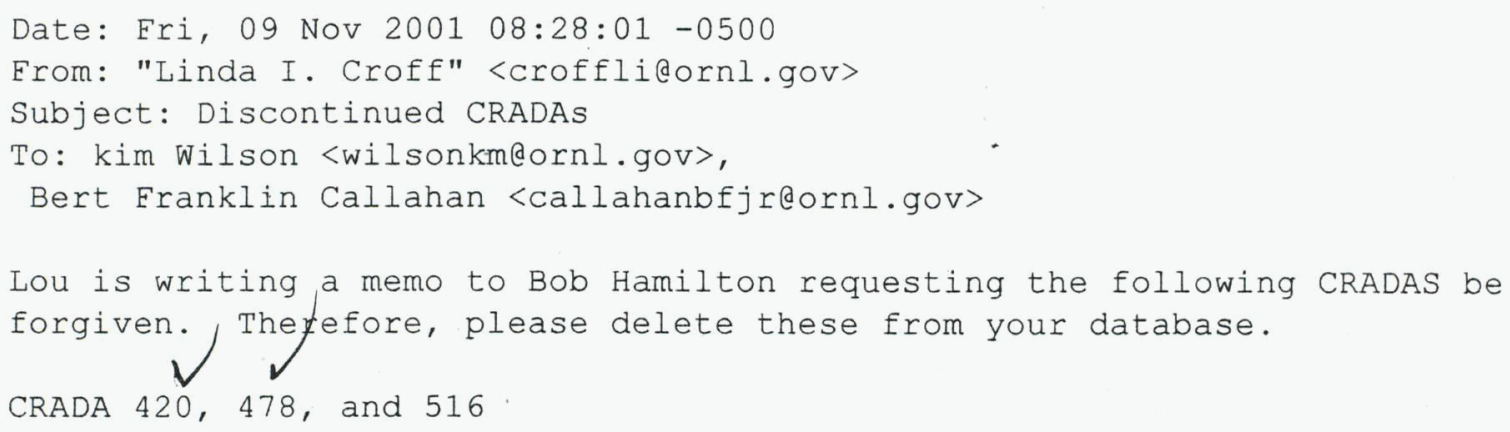

